\title{
Holographic optical elements recorded on spherical surfaces with photopolymers
}

\author{
A. Fimia, L. Carretero, and A. Beléndez
}

\begin{abstract}
A method for recording of holograms on spherical substrates is presented. As recording material we used a photopolymer placed between two spherical glass plates that are concentric.

Key words: Holography, holographic optical elements, photopolymers.
\end{abstract}

Nowadays many types of holographic optical elements (HOE's) are being used in different optical systems, and the interest for the design, fabrication, and application of these optical systems with HOE's has increased considerably. Even though HOE's offer many interesting features, aberrations are present in HOE's when the geometry and/or the wavelength are changed between recording and reconstruction. Use of spherical instead of planar substrates has been suggested as a method for reduction of both monochromatic ${ }^{1-4}$ and chromatic ${ }^{5}$ aberrations in HOE's, and the aberrations of holograms recorded on spherical surfaces have been considered in many papers..$^{2,3}$ HOE's recorded on curved surfaces have aberration characteristics different from those recorded on flat substrates. In the case of HOE's recorded on spherical surfaces an additional parameter, spherical surface radius, is available and can be used for improvement of imaging quality. However, all papers cited on curved holograms are theoretical ones, and to our knowledge experimental studies of this topic have not been presented.

In this Note, details on the possibility of fabrication of volume phase off-axis HOE's on spherical substrates and preliminary experimental results obtained are presented. Typically, different materials such as silver halide emulsion, dichromated gelatin, or photoresist are used for manufacture of HOE's. However, the serious drawback of the materials

The authors are with the Universidad de Alicante, Apartado 99, Alicante 03080, Spain; A. Beléndez is with the Departamento de Ingeniería de Sistemas y Comunicaciones, and the other authors are with the Laboratorio de Optica, Departamento Interuniversitario de Optica.

Received 30 September 1993; revised manuscript received 10 January 1994.

0003-6935/94/173633-02\$06.00/0.

(C) 1994 Optical Society of America. mentioned above is connected with relatively complex technology, especially in the creation of the photosensitive layer on a nonplane substrate. These materials also require subsequent developing, and dichromated gelatin also requires a cementing procedure. All of these problems make it difficult to experimentally analyze HOE's on spherical substrates. One possibility is to use photopolymers as a recording material for fabrication of HOE's on spherical surfaces. Photopolymers have been shown to have high sensitivity and an excellent signal-to-noise ratio. Additionally, they are usually used between two plates of glass, and because they are liquid, they conform to any type of substrate they are deposited upon. This leads us to believe that they can be used to manufacture these holographic systems and that it is possible to introduce them between two spherical plates of glass that are concentric so that by use of adequate spacers, we can generate a uniform layer of photopolymer on the spherical substrate.

Holographic photopolymer systems basically consist of a monomer, or a mixture of monomers, and a photoinitiator. The system selected here was formed by mixing of two aqueous solutions ${ }^{6,7}$ : The polymerizable system (solution A) was formed with acrylamide, $7 \mathrm{M}$; zinc acrylate, $1 \mathrm{M}$; and $N, N^{\prime}$-methylenebis-acrylamide, $0.7 \mathrm{M}$. The photoinitiator system (solution B) was formed with Methylene Blue, $3.6 \times$ $10^{-2} \mathrm{M}$; Rose Bengal, $6.0 \times 10^{-5} \mathrm{M}$; and $p$-toluensulfuric acid, $1.7 \times 10^{-2} \mathrm{M}$. The to-be-irradiated solution was formed by mixing of four volumes of solution A with one volume of solution $B$.

A set of films with the two dyes was submitted to pre-exposure at $546 \mathrm{~nm}$ and subsequent irradiation with $633 \mathrm{~nm}$. We changed the intensity and the time of pre-exposure to the beam in order to study the influence both on energetic sensitivity and the layer's rigidity. A previous paper ${ }^{6}$ showed that it is possible to increase the energetic sensitivity and at the same 


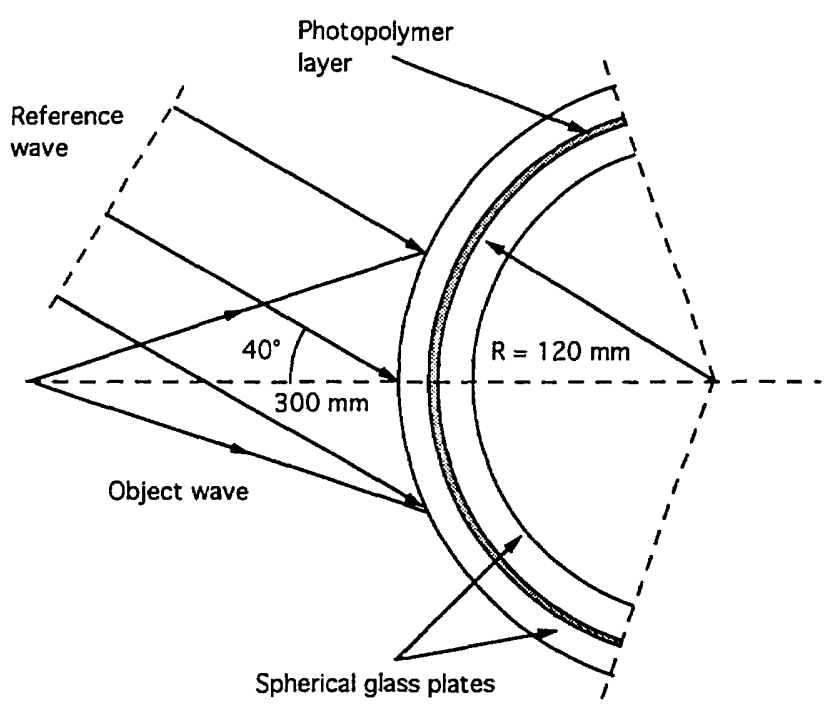

(a)

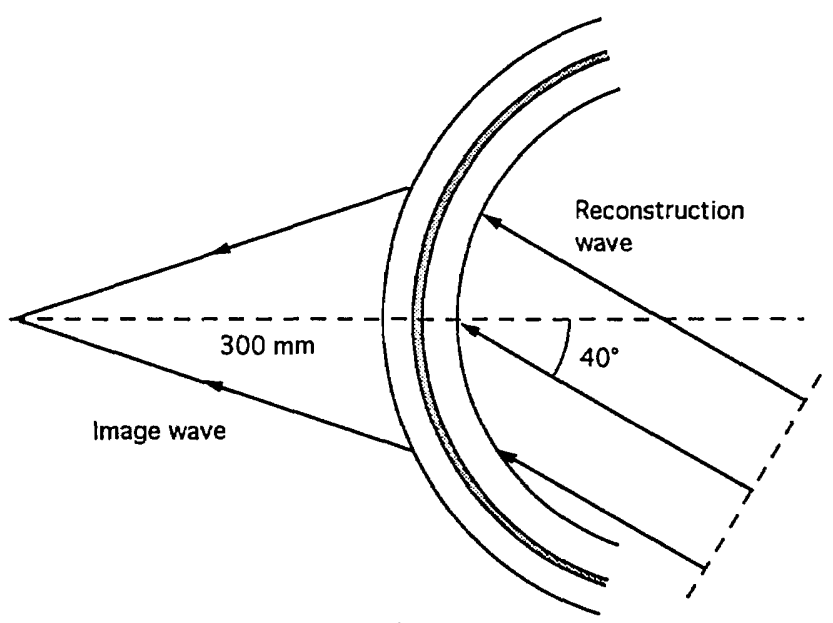

(b)

Fig. 1. Geometries of (a) recording and (b) reconstruction of the holographic optical elements.

time to have a panchromatic system. The solution is placed between two spherical glass plates with a curvature radius of $120 \mathrm{~mm}$. This device was used to make holographic lenses by means of interference of a spheric wave with a plane wave. The diameter of the usable holographic lenses was $6 \mathrm{~cm}$, and the focal length was $30 \mathrm{~cm}$. Figure 1 shows the geometries of recording and the reconstruction of the holographic lenses. The diffraction efficiency achieved with this system was $40 \%$ with a sensitivity of $5 \mathrm{~mJ} / \mathrm{cm}^{2}$ at $633 \mathrm{~nm}$, and it was $40 \%$ with a sensitivity of $12 \mathrm{~mJ} / \mathrm{cm}^{2}$ at $514 \mathrm{~nm}$. Additionally, uniformity across the holographic lens was excellent. In the future, image quality obtained by diffraction theory and system tolerances as well as the angular response of the HOE will be studied.

HOE's recorded on spheric substrates might be of great use in the design and the fabrication of head-up displays, scanners, and solar concentrators. The nonplane geometry can increase the volume response of the HOE; therefore multiplexed holographic elements can decrease its cross-talk noise. ${ }^{8}$

A portion of this research was presented at the 1993 annual meeting of the International Comission of Optics (ICO-16), Budapest, Hungary, 9-13 August 1993. This research was sponsored by the Comisión Interministerial de Ciencia y Technología, Spain (project MAT93-0369).

\section{References}

1. W. T. Weldford, "Aplanatic hologram lenses on spherical surfaces," Opt. Commun. 9, 268-269 (1973).

2. K. S. Mustafin, "Aberrations of thin holograms produced on a spherical substrate," Opt. Spectrosc. (USSR) 37, 664-666 (1974).

3. J. Masajada and J. Nowak, "Third-order aberrations for holograms and holographic lenses recorded on quadrics of revolution," Appl. Opt. 30, 1791-1795 (1991).

4. S. Baskar and K. Singh, "Imaging of extended objects using a curved holographic lens free from spherical aberration," J. Mod. Opt. 39, 1533-1542 (1992).

5. I. Weingärtner, "A holographic mirror objective," Optik 65, 49-61 (1983).

6. A. Fimia, N. López, F. Mateos, R. Sastre, J. Pineda, and F. Amat-Guerri, "Elimination of oxygen inhibition in photopolymer systems used as holographic recording materials," J. Mod. Opt. 40, 699-706 (1993).

7. A. Fimia, N. López, F. Mateos, R. Sastre, J. Pineda, and F. Amat-Guerri, "A new photopolymer useful as holographic recording material," Appl. Opt. 32, 3706-3707 (1993).

8. A. Yariv, "Interpage and interpixel cross talk in orthogonal (wavelength-multiplexed) holograms," Opt. Lett. 18, 652-654 (1993). 